PROCEEDINGS OF THE

AMERICAN MATHEMATICAL SOCIETY

Volume 134, Number 8, Pages 2363-2372

S 0002-9939(06)08403-6

Article electronically published on March 21, 2006

\title{
EQUIVALENCE OF HARDY-TYPE INEQUALITIES WITH GENERAL MEASURES ON THE CONES OF NON-NEGATIVE RESPECTIVE NON-INCREASING FUNCTIONS
}

\author{
L.-E. PERSSON, V. D. STEPANOV, AND E. P. USHAKOVA \\ (Communicated by Jonathan M. Borwein)
}

\begin{abstract}
Some Hardy-type integral inequalities in general measure spaces, where the corresponding Hardy operator is replaced by a more general Volterra type integral operator with kernel $k(x, y)$, are considered. The equivalence of such inequalities on the cones of non-negative respective non-increasing functions are established and applied.
\end{abstract}

\section{INTRODUCTION}

Let $\lambda$ and $\mu$ be regular Borel measures on $\mathbb{R}_{+}:=[0, \infty)$ such that $\lambda[0, x]<\infty$ for all $x \in \mathbb{R}_{+}, K$ is a positive operator, that is, $K f(x) \geq 0$ for any function $f(y) \geq 0$. A considerable number of works are devoted to the study of inequalities of the type

$$
\left(\int_{0}^{\infty}(K f)^{q} d \mu\right)^{1 / q} \leq C\left(\int_{0}^{\infty} f^{p} d \lambda\right)^{1 / p}
$$

where $f$ runs over a cone of non-negative functions (see, for instance, 1] and the literature given there). Let

$$
\begin{gathered}
E:=\left\{f(x) \geq 0, x \in \mathbb{R}_{+}\right\}, \\
E^{\downarrow}:=\left\{f(x) \geq 0, f(x) \text { is non-increasing for } x \in \mathbb{R}_{+}\right\}
\end{gathered}
$$

be two standard cones in the space of all $\lambda$-measurable functions. It is well known that inequality (1.1) for all $f \in E$ and the same inequality for all $f \in E^{\downarrow}$ are not equivalent in general [2]. However, as was recently discovered by G. Sinnamon [3], for the Hardy operator of the type $H f(x):=\int_{0}^{x} f d \lambda$ the equivalence takes place. We generalize this and the other results of [3] to Volterra integral operators

$$
\mathbb{K} f(x):=\int_{0}^{x} k(x, y) f(y) d \lambda(y),
$$

with a kernel $k(x, y) \geq 0$ satisfying some conditions of monotonicity.

Received by the editors March 9, 2005.

2000 Mathematics Subject Classification. Primary 26D15; Secondary 47B38.

Key words and phrases. Integral operator of the Hardy type, inequalities for monotone functions.

The work of the second and third authors was financially supported by the Russian Foundation for Basic Researches (Projects 03-01-00017 and 05-01-00422) and by the Far-Eastern Branch of the Russian Academy of Sciences (Projects 05-III-A-01-12 and 05-III- $-01-108$ ). 
Throughout the paper a relation $A \ll B$ means an inequality $A \leq c B$ with a constant $c$, depending on the parameters of summation $p$ and $q$. We note that $p^{\prime}:=p /(p-1)$ for $0<p<\infty, p \neq 1$. If $A \ll B \ll A$ or $A=c B$, we write $A \approx B$. The constants $C$ in the inequalities like (1.1) are supposed to be taken as the least possible. We assume $\int_{a}^{b}:=\int_{[a, b]}$. Uncertainties of the form $0 \cdot \infty$ are taken to be zeros. The sign : $=$ is used to determine new quantities.

\section{MAin Results}

We use the notation

$$
L^{p}(\lambda):=\left\{f \geq 0,\|f\|_{L^{p}(\lambda)}:=\left(\int_{0}^{\infty} f^{p} d \lambda\right)^{1 / p}\right\},
$$

and we need the following statement.

Proposition 2.1. If $1<p<\infty$ and a function $f(x) \geq 0$ is $\lambda$-measurable, bounded and $\operatorname{supp} f \subset \mathbb{R}_{+}$is compact, then there exists a function $f^{\circ} \in E^{\downarrow}$ such that

(i) $H f(x) \leq H f^{\circ}(x)$ for all $x>0$,

(ii) $\left\|f^{\circ}\right\|_{L^{p}(\lambda)} \leq\|f\|_{L^{p}(\lambda)}$.

Remark 2.2. The function $f^{\circ}$ is called a level function. The proof of Proposition 2.1 can be found in [4].

Theorem 2.3. Let $1 \leq p<\infty$ and $0<q<\infty$. Let the kernel $k(x, y): \mathbb{R}_{+} \times \mathbb{R}_{+} \rightarrow$ $\mathbb{R}_{+}$be non-increasing in $y \in[0, x]$ for every $x$ and let $\mathbb{K}$ be defined by (1.2). Then the inequalities

$$
\|\mathbb{K} f\|_{L^{q}(\mu)} \leq C_{1}(p, q)\|f\|_{L^{p}(\lambda)}, f \in E
$$

and

$$
\|\mathbb{K} f\|_{L^{q}(\mu)} \leq C_{2}(p, q)\|f\|_{L^{p}(\lambda)}, f \in E^{\downarrow},
$$

are equivalent and $C_{1}(p, q)=C_{2}(p, q)$.

Proof. The implication $(2.1) \Rightarrow(2.2)$ and the inequality $C_{2}(p, q) \leq C_{1}(p, q)$ are trivial because of $E^{\downarrow} \subset E$. We show the inverse. If supp $\mu=\{0\}$, then the assertion of the theorem is obvious. Therefore, let $\operatorname{supp} \mu \neq\{0\}$. By monotonicity of the kernel $k(x, y)$ we have in a sense of Stieltjes' integral that for all $0 \leq y<x<\infty$

$$
k(x, y)=k(x, x)+\int_{y}^{x} d_{z}(-k(x, z)) .
$$

By (i) of Proposition 2.1 it implies for all bounded $f \in E$ with compact support $\operatorname{supp} f \subset \mathbb{R}_{+}$that

$$
\begin{aligned}
\mathbb{K} f(x) & =k(x, x) \int_{0}^{x} f d \lambda+\int_{0}^{x}\left(\int_{y}^{x} d_{z}(-k(x, z))\right) f(y) d \lambda(y) \\
& =k(x, x) \int_{0}^{x} f d \lambda+\int_{0}^{x}\left(\int_{0}^{z} f d \lambda\right) d_{z}(-k(x, z)) \\
& \leq k(x, x) \int_{0}^{x} f^{\circ} d \lambda+\int_{0}^{x}\left(\int_{0}^{z} f^{\circ} d \lambda\right) d_{z}(-k(x, z))=\mathbb{K} f^{\circ}(x) .
\end{aligned}
$$

Applying (ii) of Proposition 2.1, we find for $1<p<\infty$ that

$$
\|\mathbb{K} f\|_{L^{q}(\mu)} \leq\left\|\mathbb{K} f^{\circ}\right\|_{L^{q}(\mu)} \leq C_{2}\left\|f^{\circ}\right\|_{L^{p}(\lambda)} \leq C_{2}\|f\|_{L^{p}(\lambda)} .
$$


For arbitrary $f \in E$ the required assertion follows by Fatou's theorem. The case $p=1$ also follows by a limiting process with $p \rightarrow 1$.

Put

$$
\Lambda(x):=\int_{0}^{x} d \lambda, \quad P_{\lambda} f(x):=\frac{1}{\Lambda(x)} H f(x) .
$$

The following statement is essentially proved in 3 .

Corollary 2.4. Let $1 \leq p<\infty, 0<q<\infty$. Then the inequalities

$$
\left\|P_{\lambda} f\right\|_{L^{q}(\mu)} \leq C_{1}(p, q)\|f\|_{L^{p}(\lambda)}, f \in E,
$$

and

$$
\left\|P_{\lambda} f\right\|_{L^{q}(\mu)} \leq C_{2}(p, q)\|f\|_{L^{p}(\lambda)}, f \in E^{\downarrow},
$$

are equivalent. Moreover, for $p>1$ (2.3) and (2.4) are equivalent to

$$
\|f\|_{L^{q}(\mu)} \leq C_{3}(p, q)\|f\|_{L^{p}(\lambda)}, f \in E^{\downarrow}
$$

and $C_{3}(p, q) \leq C_{1}(p, q)=C_{2}(p, q) \leq p^{\prime} C_{3}(p, q)$.

Proof. The proof follows from Theorem 2.3, the inequality $f(x) \leq P_{\lambda} f(x)$ for any $f \in E^{\downarrow}$ and Hardy's inequality [3]

$$
\left\|P_{\lambda} f\right\|_{L^{p}(\lambda)} \leq p^{\prime}\|f\|_{L^{p}(\lambda)}
$$

where $1<p<\infty$.

From this we obtain a sharp form of the well-known Sawyer's theorem [6. Theorem 1].

Corollary 2.5. Let $1<p<\infty$. Then

$$
S_{p}:=\sup _{f \in E^{\downarrow}} \frac{\int_{0}^{\infty} f d \mu}{\left(\int_{0}^{\infty} f^{p} d \lambda\right)^{1 / p}} \in\left[\frac{B_{p}}{p^{\prime}}, B_{p}\right],
$$

where

$$
B_{p}:=\left(\int_{0}^{\infty}\left(\int_{x}^{\infty} \frac{d \mu}{\Lambda}\right)^{p^{\prime}} d \lambda(x)\right)^{1 / p^{\prime}} .
$$

Moreover, the relation (2.7) is unimprovable in general.

Proof. It is well known [5, Chapter XI, $\S 1.5$, Theorem 4] that for $1<p<\infty, q=1$ the best constant in the inequality $(2.3)$ has the form $C_{1}(p, 1)=B_{p}$. From this and Corollary 2.4 it follows the estimate (2.7). Let us show that it is optimal. If $d \lambda(x)=\delta_{0}(x) \chi_{[0,1]}(x) d x, \operatorname{supp} \mu=[0,1]$, where $\delta_{0}(x)$ denotes the Dirac delta function at the point 0 , then it is not difficult to see that

$$
S_{p} \geq \int_{0}^{1} d \mu=B_{p}
$$

in this case. Therefore the inequality $S_{p} \leq B_{p}$ is sharp. Now, let

$$
\alpha \in\left(0,1 / p^{\prime}\right), \quad d \mu(x)=x^{-\alpha} \chi_{[0,1]}(x) d x, \quad d \lambda(x)=\chi_{[0,1]}(x) d x,
$$

where $\chi_{[0,1]}(x)$ denotes the characteristic function of the interval $[0,1]$. Then

$$
S_{p, \alpha}:=\sup _{f \in E^{\downarrow}} \frac{\int_{0}^{1} f(x) d x / x^{\alpha}}{\left(\int_{0}^{1} f^{p}(x) d x\right)^{1 / p}}=\left(1-\alpha p^{\prime}\right)^{-1 / p^{\prime}} .
$$


Here the upper bound follows from the Hölder inequality, and the lower bound is achieved for the function $f(x)=x^{-\alpha /(p-1)}$. We also have

$$
B_{p, \alpha}:=\left(\int_{0}^{1}\left(\int_{x}^{1} \frac{d y}{y^{1+\alpha}}\right)^{p^{\prime}} d x\right)^{1 / p^{\prime}}=\frac{1}{\alpha}\left(\int_{0}^{1}\left(1-x^{\alpha}\right)^{p^{\prime}} \frac{d x}{x^{\alpha p^{\prime}}}\right)^{1 / p^{\prime}} .
$$

Applying the elementary inequality

$$
\left(1-x^{\alpha}\right)^{p^{\prime}} \geq 1-p^{\prime} x^{\alpha}
$$

we find

$$
B_{p, \alpha} \geq \frac{1}{\alpha}\left(\int_{0}^{1}\left(1-p^{\prime} x^{\alpha}\right) \frac{d x}{x^{\alpha p^{\prime}}}\right)^{1 / p^{\prime}}=\frac{1}{\alpha}\left(1-p^{\prime} \alpha\right)^{-1 / p^{\prime}}\left(1-\frac{p^{\prime}\left(1-p^{\prime} \alpha\right)}{1-\alpha\left(p^{\prime}-1\right)}\right)^{1 / p^{\prime}} .
$$

Hence,

$$
\lim _{\alpha \rightarrow 1 / p^{\prime}} \frac{S_{p, \alpha}}{B_{p, \alpha}} \leq \frac{1}{p^{\prime}}
$$

and, consequently, the estimate $p^{\prime} S_{p} \geq B_{p}$ is sharp, too.

Theorem 2.6. Let $1<p<\infty, 0<q<\infty$ and let $m, n$ be any non-negative integers. Then the inequalities (2.1), (2.2)

and

$$
\left\|\left(\mathbb{K} P_{\lambda}^{n}\right) f\right\|_{L^{q}(\mu)} \leq C_{4}\|f\|_{L^{p}(\lambda)}, f \in E,
$$

are mutually equivalent.

Proof. If $f \in E^{\downarrow}$, then $f \leq P_{\lambda} f \leq \ldots \leq P_{\lambda}^{n} f$ and (2.9) implies (2.2) by

$$
\|K f\|_{L^{q}(\mu)} \leq\left\|K P_{\lambda}^{n} f\right\|_{L^{q}(\mu)} \leq C_{5}\left\|P_{\lambda}^{m} f\right\|_{L^{p}(\lambda)} \leq C_{5}\left(p^{\prime}\right)^{m}\|f\|_{L^{p}(\lambda)} .
$$

Now, $(2.2) \Rightarrow(2.1)$ follows by Theorem 2.3 and (2.1) $\Rightarrow(2.8)$ by

$$
\left\|K P_{\lambda}^{n} f\right\|_{L^{q}(\mu)} \leq C_{1}\left\|P_{\lambda}^{n} f\right\|_{L^{p}(\lambda)} \leq C_{1}\left(p^{\prime}\right)^{n}\|f\|_{L^{p}(\lambda)} .
$$

Finally, if $f \in E^{\downarrow}$, then again applying $f \leq P_{\lambda} f \leq \ldots \leq P_{\lambda}^{m} f$ we see that $(2.8) \Rightarrow$ (2.9) follows by

$$
\left\|K P_{\lambda}^{n} f\right\|_{L^{q}(\mu)} \leq C_{4}\|f\|_{L^{p}(\lambda)} \leq C_{4}\left\|P_{\lambda}^{m} f\right\|_{L^{p}(\lambda)} .
$$

In the theory of the weighted Hardy-type inequalities the following is well known.

Definition 2.7. A kernel $k(x, y) \geq 0$ of the integral operator of the form (1.2) belongs to Oinarov's class, $k \in \mathcal{O}$, if there is a constant $D \geq 1$ such that

$$
D^{-1} k(x, y) \leq k(x, z)+k(z, y) \leq D k(x, y), x \geq z \geq y \geq 0 .
$$

Remark 2.8. It is known [7] that if $k \in \mathcal{O}$, then

$$
k(x, y) \leq \bar{k}(x, y) \leq D k(x, y),
$$

where

$$
\bar{k}(x, y)=\sup _{z \in[y, x]} k(x, z) .
$$

Therefore, Theorem 2.6 is valid for integral operators $\mathbb{K}$ of the form (1.2) with a kernel $k \in \mathcal{O}$, because $\bar{k}(x, y)$ is non-increasing in $y \in[0, x]$. 
Put

$$
\begin{gathered}
K_{\lambda}(x):=\int_{0}^{x} k(x, y) d \lambda(y), \\
P_{K} f(x):=\frac{1}{K_{\lambda}(x)} \int_{0}^{x} k(x, y) f(y) d \lambda(y) .
\end{gathered}
$$

Definition 2.9. We write that a kernel $k(x, y) \in \mathcal{D}_{\lambda}$ if $P_{K} f \in E^{\downarrow}$ for any $f \in E^{\downarrow}$.

Observe that the class $D_{\lambda}$ consists of non-negative kernels $k(x, y)$ such that for all $t>0$

$$
\frac{1}{K_{\lambda}(x)} \int_{0}^{t} k(x, y) d \lambda(y)
$$

is non-increasing in $x$ when $x \geq t$. Necessity follows from the inequality $P_{K} f(x) \geq$ $P_{K} f(z)$ with $x \geq z \geq t$ and $f=\chi_{[0, t]}$. For sufficiency we assume that $f(x)=\int_{x}^{\infty} h$, $h \geq 0$. Then for $x \geq t$ we have

$$
\begin{aligned}
P_{K} f(x) & =f(x)+\int_{t}^{x} h(s) \frac{\int_{0}^{s} k(x, y) d \lambda(y)}{K_{\lambda}(x)} d s \\
& +\int_{0}^{t} h(s) \frac{\int_{0}^{s} k(x, y) d \lambda(y)}{K_{\lambda}(x)} d s \\
& \geq \int_{t}^{\infty} h+\int_{0}^{t} h(s) \frac{\int_{0}^{s} k(t, y) d \lambda(y)}{K_{\lambda}(t)} d s=P_{K} f(t) .
\end{aligned}
$$

Now we intend to obtain the equivalence like $(2.3) \Leftrightarrow(2.5)$ for the more general inequality (2.1) instead of (2.3). To this end we need an analog of the Hardy inequality $(2.6)$ for the operator $P_{K}($ see $[8, \S 2.3])$.

Proposition 2.10. Let $1<p<\infty$ and $k(x, y) \in \mathcal{O}$. Then

$$
\left\|P_{K} f\right\|_{L^{p}(\lambda)} \leq C_{6}\|f\|_{L^{p}(\lambda)}, f \in E,
$$

if and only if

$$
\mathbb{A}_{0}:=\sup _{t \in \mathbb{R}_{+}} \frac{\left(\int_{0}^{t}\left(\frac{1}{K_{\lambda}(x)} \int_{0}^{x}[k(x, y)]^{p^{\prime}} d \lambda(y)\right)^{p} d \lambda(x)\right)^{1 / p}}{\left(\int_{0}^{t}[k(t, z)]^{p^{\prime}} d \lambda(z)\right)^{1 / p}}<\infty
$$

and $\mathbb{A}_{0} \approx C_{6}$.

Proof. Necessity. For any $t>0$ put $f_{t}(y)=[k(t, y)]^{p^{\prime}-1} \chi_{[0, t]}(y)$. Then (2.13) with $f=f_{t}$ gives

$$
\begin{aligned}
C_{6}^{p} \int_{0}^{t}[k(t, z)]^{p^{\prime}} d \lambda(z) & \geq \int_{0}^{t}\left(\frac{1}{K_{\lambda}(x)} \int_{0}^{x}[k(t, y)]^{p^{\prime}-1} k(x, y) d \lambda(y)\right)^{p} d \lambda(x) \\
& \geq D^{-p^{\prime}} \int_{0}^{t}\left(\frac{1}{K_{\lambda}(x)} \int_{0}^{x}[k(x, y)]^{p^{\prime}} d \lambda(y)\right)^{p} d \lambda(x) .
\end{aligned}
$$

Hence, $D^{p^{\prime} / p} C_{6} \geq \mathbb{A}_{0}$.

For the sufficiency we observe that (2.13) is equivalent to

$$
\left\|P_{K}^{*} g\right\|_{L^{p^{\prime}}(\lambda)} \leq C_{6}\|g\|_{L^{p^{\prime}}(\lambda)}, g \in E,
$$


where

$$
P_{K}^{*} g(y):=\int_{y}^{\infty} k(x, y) g(x) \frac{d \lambda(x)}{K_{\lambda}(x)} .
$$

Now, applying [8, Lemma 2], whose proof can be duplicated for a general measure, we find that

$$
\begin{aligned}
\left\|P_{K}^{*} g\right\|_{L^{p^{\prime}(\lambda)}}^{p^{\prime}} & \ll \int_{0}^{\infty} g\left(P_{K}^{*} g\right)^{p^{\prime}-1} d \lambda \\
& +\int_{0}^{\infty} g(y)\left(\int_{y}^{\infty} g(z) \frac{d \lambda(z)}{K_{\lambda}(z)}\right)^{p^{\prime}-1}\left(\int_{0}^{y}[k(y, s)]^{p^{\prime}} d \lambda(s)\right) \frac{d \lambda(y)}{K_{\lambda}(y)} \\
& =: J_{1}+J_{2} .
\end{aligned}
$$

Assume that $g$ is bounded with compact support and $0<\left\|P_{K}^{*} g\right\|_{L^{p^{\prime}}(\lambda)}<\infty$. By Hölder's inequality we have

$$
J_{1} \leq\|g\|_{L^{p^{\prime}(\lambda)}}\left\|P_{K}^{*} g\right\|_{L^{p^{\prime}(\lambda)}}^{p^{\prime} / p}
$$

Again by Hölder's inequality we find

$$
\begin{aligned}
J_{2} & \leq\|g\|_{L^{p^{\prime}}(\lambda)}\left(\int_{0}^{\infty}\left(\int_{y}^{\infty} g(z) \frac{d \lambda(z)}{K_{\lambda}(z)}\right)^{p^{\prime}}\left(\frac{1}{K_{\lambda}(y)} \int_{0}^{y}[k(y, s)]^{p^{\prime}} d \lambda(s)\right)^{p} d \lambda(y)\right)^{1 / p} \\
& =:\|g\|_{L^{p^{\prime}(\lambda)}} J_{2,1}^{1 / p} .
\end{aligned}
$$

Now, once more applying the measure version of [8, Lemma 2] we obtain

$$
\begin{aligned}
J_{2,1} & \ll \int_{0}^{\infty} g(z) \frac{d \lambda(z)}{K_{\lambda}(z)}\left(\int_{z}^{\infty} g(x) \frac{d \lambda(x)}{K_{\lambda}(x)}\right)^{p^{\prime}-1} \int_{0}^{z}\left(\frac{1}{K_{\lambda}(y)} \int_{0}^{y}[k(y, s)]^{p^{\prime}} d \lambda(s)\right)^{p} d \lambda(y) \\
& \leq \mathbb{A}_{0}^{p} J_{2} .
\end{aligned}
$$

Hence, $J_{2} \ll \mathbb{A}_{0}^{p^{\prime}}\|g\|_{L^{p^{\prime}(\lambda)}}^{p^{\prime}}$. Thus,

$$
\left\|P_{K}^{*} g\right\|_{L^{p^{\prime}(\lambda)}}^{p^{\prime}} \ll\|g\|_{L^{p^{\prime}(\lambda)}}\left\|P_{K}^{*} g\right\|_{L^{p^{\prime}(\lambda)}}^{p^{\prime} / p}+\mathbb{A}_{0}^{p^{\prime}}\|g\|_{L^{p^{\prime}(\lambda)}}^{p^{\prime}},
$$

and the required upper bound follows.

Theorem 2.11. Let $1<p<\infty, 0<q<\infty$ and let a kernel $k \in \mathcal{O} \cap \mathcal{D}_{\lambda}$ be such that $\mathbb{A}_{0}<\infty$. Then the inequality (2.1) is equivalent to

$$
\left\|f K_{\lambda}\right\|_{L^{q}(\mu)} \leq C_{7}\|f\|_{L^{p}(\lambda)}, f \in E^{\downarrow}
$$

and $C_{1} \approx C_{7}$.

Proof. First we show that $(2.14) \Rightarrow(2.2)$. Let $f \in E^{\downarrow}$. Then $P_{K} f \in E^{\downarrow}$, because of $k \in \mathcal{D}_{\lambda}$. Therefore

$$
\|K f\|_{L^{q}(\mu)}=\left\|\left(P_{K} f\right) K_{\lambda}\right\|_{L^{q}(\mu)} \leq C_{7}\left\|P_{K} f\right\|_{L^{p}(\lambda)},
$$

and by the inequality $(2.13)$

$$
\ll C_{7} \mathbb{A}_{0}\|f\|_{L^{p}(\lambda)} \text {. }
$$

In view of Remark 2.8 this implies (2.1) and, moreover, $C_{1} \ll C_{7} \mathbb{A}_{0}$. Conversely, if $f \in E^{\downarrow}$, then by $(2.11)$

$$
K_{\lambda}(x) \leq \bar{K}_{\lambda}(x):=\int_{0}^{x} \bar{k}(x, y) d \lambda(y) .
$$


Therefore,

$$
P_{K} f(x) \geq \frac{1}{D \bar{K}_{\lambda}(x)} \int_{0}^{x} \bar{k}(x, y) f(y) d \lambda(y) \geq \frac{1}{D} f(x) .
$$

Thus, if (2.1) is true, then for $f \in E^{\downarrow}$ we have

$$
\left\|f K_{\lambda}\right\|_{L^{q}(\mu)} \leq D\|K f\|_{L^{q}(\mu)} \leq D C_{1}\|f\|_{L^{p}(\lambda)} .
$$

Consequently, $C_{7} \leq D C_{1}$.

Combining Theorems 2.6 and 2.11, we obtain the following

Corollary 2.12. Let $1<p<\infty, 0<q<\infty$ and let a kernel $k \in \mathcal{O} \cap \mathcal{D}_{\lambda}$ be such that $\mathbb{A}_{0}<\infty$. Then (2.14) is equivalent to (2.8) and to (2.9).

Remark 2.13. The condition $k \in \mathcal{D}_{\lambda}$ was not used in the proof of $(2.1) \Rightarrow(2.14)$, and therefore (2.14) follows either from (2.1), (2.2), (2.8) or (2.9), whenever $k \in \mathcal{O}$ and $\mathbb{A}_{0}<\infty$.

Let $\varphi:(0, \infty) \rightarrow(-\infty, \infty)$ be such a monotone function so that there exists the reverse function $\varphi^{-1}$ and either

(a) $\varphi$ is concave and increasing

or

(b) $\varphi$ is convex and decreasing.

Put

$$
\Phi_{K} f(x):=\varphi^{-1}\left[P_{K}(\varphi(f))\right](x)=\varphi^{-1}\left[\frac{1}{K_{\lambda}(x)} \int_{0}^{x} k(x, y)[\varphi(f)](y) d \lambda(y)\right] .
$$

Our following assertion generalizes the result of G. Sinnamon [3, Theorem 4.1], established for $k(x, y)=1$.

Theorem 2.14. Let $1<p<\infty, 0<q<\infty, k \in \mathcal{O} \cap \mathcal{D}_{\lambda}$ and $\mathbb{A}_{0}<\infty$. Suppose that $\varphi$ satisfies the condition (a) or (b). Then the following inequalities are equivalent:

$$
\begin{gathered}
\|f\|_{L^{q}(\mu)} \leq C_{8}\|f\|_{L^{p}(\lambda)}, f \in E^{\downarrow}, \\
\left\|P_{K} f\right\|_{L^{q}(\mu)} \leq C_{9}\|f\|_{L^{p}(\lambda)}, f \in E^{\downarrow}, \\
\left\|P_{K} f\right\|_{L^{q}(\mu)} \leq C_{10}\|f\|_{L^{p}(\lambda)}, f \in E, \\
\left\|\Phi_{K} f\right\|_{L^{q}(\mu)} \leq C_{11}\|f\|_{L^{p}(\lambda)}, f \in E, \\
\left\|\Phi_{K} f\right\|_{L^{q}(\mu)} \leq C_{12}\|f\|_{L^{p}(\lambda)}, f \in E^{\downarrow} .
\end{gathered}
$$

Moreover, the least possible constants in the inequalities (2.15)-(2.19) are also pairwise equivalent.

Proof. (2.15) $\Rightarrow(2.16) \Rightarrow(2.17)$ follows from Theorems 2.3 and 2.11 and Remark 2.8, and $(2.17) \Rightarrow(2.18)$ follows by applying Jensen's inequality $\Phi_{K} f(x) \leq P_{K} f(x)$. $(2.18) \Rightarrow(2.19)$ is trivial. It is left to show that $(2.19) \Rightarrow(2.15)$. Let $f \in E^{\downarrow}$ and $\varphi$ satisfy condition (a). Then $\varphi(f)$ is non-increasing. This easily implies that $\varphi(f)(x) \leq P_{K}(\varphi(f))(x)$. Applying $\varphi^{-1}$ to the both parts of this inequality, we conclude that $f(x) \leq \Phi_{K} f(x)$ and therefore, $(2.19) \Rightarrow(2.15)$; moreover, $C_{8} \leq C_{12}$. If $\varphi$ satisfies condition (b) and $f \in E^{\downarrow}$, then $\varphi(f)$ is non-decreasing. Then $\varphi(f)(x) \geq P_{K}(\varphi(f))(x)$. Again applying $\varphi^{-1}$ to the both parts of this inequality, we obtain that $f(x) \leq \Phi_{K} f(x)$ and therefore $(2.19) \Rightarrow(2.15)$ and $C_{8} \leq C_{12}$. 
Remark 2.15. Until now we have mainly studied only the equivalence of the inequalities, and it may seem that their own characterization is still open. However, this is not so, because the characterization of the inequality (2.15) is well known (see [9, Proposition 1] for weighted inequalities and [3] for inequalities with general measures). Namely, for $0<p \leq q<\infty$

$$
C_{8}(p, q)=\sup _{f \in E \downarrow} \frac{\left(\int_{0}^{\infty} f^{q} d \mu\right)^{1 / q}}{\left(\int_{0}^{\infty} f^{p} d \lambda\right)^{1 / p}}=\sup _{t>0} \frac{\left(\int_{0}^{t} d \mu\right)^{1 / q}}{\left(\int_{0}^{t} d \lambda\right)^{1 / p}}
$$

and for $0<q<p<\infty, 1 / r:=1 / q-1 / p$

$$
C_{8}(p, q) \in\left[\frac{q}{r} B_{p / q}^{1 / q}, B_{p / q}^{1 / q}\right]
$$

where

$$
B_{p / q}^{1 / q}=\left(\int_{0}^{\infty}\left(\int_{x}^{\infty} \frac{d \mu}{\Lambda}\right)^{r / q} d \lambda(x)\right)^{1 / r} .
$$

Observe that (2.21) follows by the change $f^{q} \rightarrow f$ in the middle term (2.20) and applying (2.7). Thus, the borders for the constant $C_{8}(p, q)$ in $(2.21)$ are sharp and the inequalities $(2.16)-(2.19)$ are characterized by finiteness of the right-hand side of (2.20) for $1<p \leq q<\infty$ and by finiteness of the right-hand side of (2.22) for $0<q<p<\infty, p>1$.

Also note that under some additional restriction on the measure $\lambda$ there is an alternative two-sided estimate of the constant $C_{8}(p, q)$ for $0<q<p<\infty$, also useful in applications. This restriction on the measure $\lambda$ was studied in 3 and always holds, for example, if the measure $\lambda$ is non-atomic. For the absolutely continuous measures $\lambda$ and $\mu$ this alternative estimate was pointed out in [10, formula (1.6)].

Finally, we will point out two applications of Theorem 2.14 , found for $k(x, y) \equiv 1$ and $f \in E$ in 3 .

Proposition 2.16. Let $0<p, q<\infty, k \in \mathcal{O} \cap \mathcal{D}_{\lambda}$ and $\mathbb{A}_{0}<\infty$. Then the inequality

$$
\left(\int_{0}^{\infty} f^{-p} d \lambda\right)^{-1 / p} \leq C_{13}\left(\int_{0}^{\infty}\left(P_{K} f\right)^{-q} d \mu\right)^{-1 / q}
$$

for all $f \in E$ is also equivalent to the same inequality for all $f \in E^{\downarrow}$ and equivalent to the inequality (2.15). Moreover, $C_{13} \approx C_{8}$.

Proof. If $0<q<\infty, p>1$, then applying Theorem 2.14 with $\varphi(x)=1 / x$, we obtain the required assertion and $C_{13} \approx C_{8}$. If $0<p \leq 1$, then by the change $f^{p}=g^{2}$ in the inequality (2.15) it is equivalently reproduced in the form

$$
\left(\int_{0}^{\infty} g^{2 q / p} d \mu\right)^{p / 2 q} \leq C_{8}^{p / 2}\left(\int_{0}^{\infty} g^{2} d \lambda\right)^{1 / 2}, g \in E^{\downarrow} .
$$

Moreover, by Theorem 2.14 with $\varphi(x)=x^{-2 / p}$, the inequality $(2.24)$ is equivalent to the inequality

$$
\left(\int_{0}^{\infty}\left(P_{K} g^{-2 / p}\right)^{-q} d \mu\right)^{p / 2 q} \leq C_{8}^{p / 2}\left(\int_{0}^{\infty} g^{2} d \lambda\right)^{1 / 2}
$$


for all $f \in E$ or for all $f \in E^{\downarrow}$. Performing in (2.25) the reverse change $g^{-2 / p}=f$, we arrive at the inequality (2.23).

By a similar method we obtain the following.

Proposition 2.17. Let $0<p, q<\infty, k \in \mathcal{O} \cap \mathcal{D}_{\lambda}$ and $\mathbb{A}_{0}<\infty$. Then the inequalities

$$
\left(\int_{0}^{\infty}\left(\exp \left[P_{K}(\log f)\right]\right)^{q} d \mu\right)^{1 / q} \leq C_{14}\left(\int_{0}^{\infty} f^{p} d \lambda\right)^{1 / p}
$$

and

$$
\left(\int_{0}^{\infty} f^{-p} d \lambda\right)^{-1 / p} \leq C_{15}\left(\int_{0}^{\infty}\left(\exp \left[P_{K}(\log f)\right]\right)^{-q} d \mu\right)^{-1 / q}
$$

either for all $f \in E$ or for all $f \in E^{\downarrow}$ are equivalent to each other and also equivalent to the inequality (2.15). Moreover, $C_{14} \approx C_{15} \approx C_{8}$.

Proof. The inequalities (2.26) and (2.27) for $0<q<\infty, p>1$ follow from Theorem 2.14 with $\varphi(x)=\log x$, and $\varphi(x)=\log x^{-1}$, respectively. For $0<p \leq 1$ we use the arguments from the proof of Proposition 2.16 with the functions $\varphi(x)=\log x^{2 / p}$ and $\varphi(x)=\log x^{-2 / p}$.

Remark 2.18. Proposition 2.16 supplements the results of the Prokhorov recent paper [11, and Proposition 2.17 supplements the results of the papers [12], [13].

\section{ACKNOWLEDGEMENT}

The authors express their sincere gratitude to the referee for valuable comments.

\section{REFERENCES}

1. A. Kufner and L.E. Persson, Weighted inequalities of Hardy type. World Scientific Publishing Co., Singapore/New Jersey/London/Hong Kong, 2003. MR1982932 (2004c:42034)

2. M. Arino and B. Muckenhoupt, Maximal functions on classical Lorentz spaces and Hardy's inequality with weights for non-increasing functions, Trans. Amer. Math. Soc. (2) 320 (1990), 727-735. MR0989570 (90k:42034)

3. G. Sinnamon, Hardy's inequality and monotonocity, In: Function Spaces, Differential Operators and Nonlinear Analysis (Eds.: P. Drábec and J. Rákosnik), Mathematical Institute of the Academy of Sciences of the Czech Republic, Prague 2005, 292-310.

4. G. Sinnamon, Spaces defined by the level function and their duals, Studia Math. 111 (1994), 19-52. MR 1292851 (95k:46043)

5. L.V. Kantorovich and G.P. Akilov, Functional Analysis. Pergamon Press, Oxford, 1982. MR0664597 (83h:46002)

6. E. Sawyer, Boundedness of classical operators on classical Lorentz spaces, Studia Math. 96 (1990), 145-158. MR 1052631 (91d:26026)

7. E. Lomakina and V. Stepanov, On the Hardy-type integral operators in Banach function spaces, Publ. Mat. 42 (1998), 165-194. MR.1628166 (99g:47070)

8. V.D. Stepanov, Weighted norm inequalities of Hardy type for a class of integral operators, J. London Math. Soc. 50 (1994), 105-120. MR1277757 (95h:47047)

9. V.D. Stepanov, The weighted Hardy's inequality for nonincreasing functions, Trans. Amer. Math. Soc. (1) 338 (1993), 173-186. MR1097171 (93j:26012)

10. M.L. Goldman, H.P. Heinig and V.D. Stepanov, On the principle of duality in Lorentz spaces, Canad. J. Math. (5) 48 (1996), 959-979. MR1414066 (97h:42008)

11. D.V. Prokhorov, Weighted Hardy's inequalities for negative indices, Publ. Mat. 48 (2004), 423-443. MR2091014 
12. L.-E. Persson and V.D. Stepanov, Weighted integral inequalities with the geometric mean operator, J. Ineq. Appl. (5) 7 (2002), 727-746. MR1931263 (2003g:26028)

13. M.G. Nassyrova, L.-E. Persson and V.D. Stepanov, On weighted inequalities with geometric mean operator generated the Hardy-type integral transform, J. Ineq. Pure Appl. Math. (4) 3 (2002) Art. 48, 1-16. MR 1923347 (2003j:26021)

Department of Mathematics, Lulea University of Technology, SE-97187 Lulea, SweDEN

E-mail address: larserik@sm.luth.se

Department of Mathematical Analysis, Peoples Friendship University of Russia, Miklukho-Maklay 6, Moscow, 117198, Russia

E-mail address: vstepanov@sci.pfu.edu.ru

Computer Center of FEB Ras, Tikhookeanskaya 153, Khabarovsk, 680042, Russia

E-mail address: ushakova@as.khb.ru 